\title{
Benefits of prophylactic continuous infusion of furosemide after the maze procedure for atrial fibrillation
}

Niv Ad, MDa

Johan P. Suyderhoud, MD

Young D. Kim, MD

Martin A. Makary, MD, MPH

Kerry W. DeGroot, MD

Henry C. Lue, MD

Eugen A. Pirovic, $M^{b}$

Weijia Z. Duvall, $\mathrm{MD}^{\mathrm{b}}$

James L. Cox, MDa

From the Department of Thoracic and Cardiovascular Surgery ${ }^{\mathrm{a}}$ and the Division of Cardiac Anesthesiology, ${ }^{\mathrm{b}}$ Georgetown University Medical Center, Washington, DC.

Received for publication May 16, 2001; revisions requested June 26, 2001; revisions received July 16, 2001; accepted for publication July 23, 2001.

Address for reprints: Niv Ad, MD, Cardiothoracic Surgery Department, Hadassah University Hospital, Jerusalem 91120, Israel (E-mail: nivadmd@hotmail.com).

J Thorac Cardiovasc Surg 2002;123:232-6

Copyright (C) 2002 by The American Association for Thoracic Surgery

$0022-5223 / 2002 \$ 35.00+0 \quad \mathbf{1 2 / 1 / 1 1 9 0 5 8}$

doi: $10.1067 / \mathrm{mtc} .2002 .119058$
Objectives: One of the most significant complications seen after the maze procedure for atrial fibrillation is excessive fluid retention, with subsequent pulmonary complications. To address this problem we recently started treating all patients prophylactically with a continuous infusion of furosemide starting immediately after the operation.

Methods: Seventy-five consecutive patients with statistically similar demographic characteristics were divided into two groups. In the continuous infusion group $(\mathrm{n}=$ 36) furosemide was given intravenously as a continuous infusion at a dose of 2 to $15 \mathrm{mg} / \mathrm{h}$ for the first 48 hours after the operation, and in the bolus dose group $(\mathrm{n}=$ 39) furosemide was administered in bolus doses (50-100 mg) to maintain a targeted daily urinary output of 25 to $50 \mathrm{~mL} / \mathrm{kg}$. Hemodynamic data, urinary output, fluid balance, daily weights, and pulmonary complications were recorded during the first 48 hours after the operation.

Results: The mean postoperative urinary output was higher, the total furosemide dose was lower, and the pulmonary complications were fewer in the continuous infusion group than in the bolus dose group. Three patients in the bolus dose group were reintubated after the operation, whereas none in the continuous infusion group were reintubated. Supplemental oxygen requirements were greater in the bolus dose group ( 7 vs 4 patients, $P<.05$ ). In the bolus dose group, 4 patients $(10 \%)$ required thoracentesis and 3 patients $(8 \%)$ required chest tube insertions for postoperative pleural effusions, in contrast with 1 patient $(3 \%)$ each in the continuous infusion group $(P<.05)$.

Conclusion: Despite a smaller total dose relative to bolus infusion, prophylactic continuous furosemide infusion decreased the adverse pulmonary complications associated with excessive fluid retention in patients undergoing the maze procedure for atrial fibrillation.

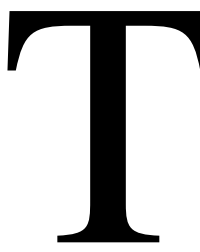

he maze procedure has become a safe and effective surgical treatment for medically refractory atrial fibrillation. ${ }^{1,2}$ Our experience and that of others has revealed that after the maze procedure patients tend to retain large amounts of fluid, which may lead to symptomatic pleural effusion and pulmonary edema. The fluid retention phenomenon occurs in as many as $30 \%$ of patients after the maze procedure..$^{2,3}$ The suggested mechanism is the decreased secretion of atrial natriuretic peptide (ANP) by atrial tissue with subsequent abrupt reduction in circulating 
TABLE 1. Patient data

\begin{tabular}{|c|c|c|c|c|}
\hline & Total $(n=75)$ & $\begin{array}{l}\text { Continuous infusion group } \\
\qquad(\mathrm{n}=\mathbf{3 6})\end{array}$ & $\begin{array}{l}\text { Bolus dose group } \\
\qquad(\mathrm{n}=39)\end{array}$ & $P$ value \\
\hline \multicolumn{5}{|l|}{ Age (y) } \\
\hline Mean & 52 & 52 & 51 & \\
\hline Range & $24-77$ & $33-72$ & $24-77$ & $>.05$ \\
\hline \multicolumn{5}{|l|}{ Male } \\
\hline No. & 55 & 28 & 27 & \\
\hline$\%$ & $73 \%$ & $77 \%$ & $69 \%$ & $>.05$ \\
\hline \multicolumn{5}{|l|}{ Procedure type (No.) } \\
\hline Maze procedure only & 49 & 24 & 25 & $>.05$ \\
\hline Maze procedure and other procedure & 26 & 12 & 14 & $>.05$ \\
\hline Minimally invasive maze procedure & 30 & 18 & 12 & $<.05$ \\
\hline Cardiopulmonary bypass time (min) & $163.6 \pm 36.3$ & $147.0 \pm 22.0$ & $179.0 \pm 40.2$ & $>.05$ \\
\hline Crossclamp time (min) & $107.4 \pm 29.6$ & $97.5 \pm 20.7$ & $116.5 \pm 33.7$ & $>.05$ \\
\hline Baseline creatinine (mg/dL) & & $1.03 \pm 0.11$ & $1.02 \pm 0.08$ & $>.05$ \\
\hline Postoperative cardiac index $\left(\mathrm{L} \cdot \mathrm{min}^{-1} \cdot \mathrm{m}^{-2}\right)$ & & $2.53 \pm 0.27$ & $2.67 \pm 0.29$ & $>.05$ \\
\hline
\end{tabular}

TABLE 2. Pulmonary complications

\begin{tabular}{|c|c|c|c|c|c|}
\hline & \multicolumn{2}{|c|}{ Continuous infusion group ( $n=36$ ) } & \multicolumn{2}{|c|}{ Bolus dose group ( $\mathrm{n}=39$ ) } & \multirow[b]{2}{*}{$P$ value } \\
\hline & No. & $\%$ & No. & $\%$ & \\
\hline \multicolumn{6}{|l|}{ Pleural effusion } \\
\hline Small & 2 & 5.5 & 6 & 15 & $<.05$ \\
\hline Medium & 1 & 3 & 4 & 10 & $<.05$ \\
\hline Large & 1 & 3 & 3 & 8 & $<.05$ \\
\hline \multicolumn{6}{|l|}{ Pulmonary edema } \\
\hline Oxygen supplementation & 4 & 10 & 0 & 0 & $>.05$ \\
\hline Reintubation & 7 & 18 & 3 & 39 & $<.05$ \\
\hline
\end{tabular}

ANP. ${ }^{2,4}$ Other studies have supported this finding by demonstrating a significant decrease in ANP levels in the plasma after successful nonsurgical cardioversion. ${ }^{5,6} \mathrm{We}$ have also demonstrated that patients who have undergone the maze procedure have significantly higher levels of systemic arginine vasopressin and aldosterone than those of patients who have undergone coronary artery bypass grafting. Both mechanisms may contribute to fluid retention after the maze procedure.

Several studies have demonstrated that continuous furosemide infusion results in a greater diuresis than is seen with bolus administration of the drug. ${ }^{6-12}$ In an effort to minimize the risk of fluid retention and its associated complication, we therefore recently tested a new protocol for diuresis management of patients after the maze procedure. In this regimen we treat patients with a continuous infusion of furosemide starting at the time of admission to the intensive care unit immediately after the operation and for 48 hours thereafter. This article reports the effects of continuous furosemide infusion on fluid balance in patients after the maze procedure, including the effect of such treatment on the incidence of pulmonary complications.

\section{Methods}

Between June 1997 and January 1999 we performed 90 maze procedures. Fifty-two patients had the maze procedure as the only operation and 38 patients had a total of 60 different procedures combined with the maze procedure. Ten patients had coronary artery bypass grafting, 37 had mitral valve operations (19 patients underwent mitral valve repair), 3 patients had tricuspid valve repair, 6 patients had aortic valve replacement, and 4 patients had the maze procedure combined with repair of congenital anomalies. In 30 cases the maze procedure was performed with a minimally invasive approach; however, all patients in this study underwent the maze III procedure, without the amputation of both right and left atrial appendages.

Fifteen patients were excluded from the study for the following reasons: 7 patients ( 3 in the continuous intravenous infusion group) had preoperative creatinine levels higher than $1.5 \mathrm{mg} / \mathrm{dL}$, and 8 patients ( 3 in the continuous intravenous infusion group) had hemodynamic instability requiring significant inotropic support. The 


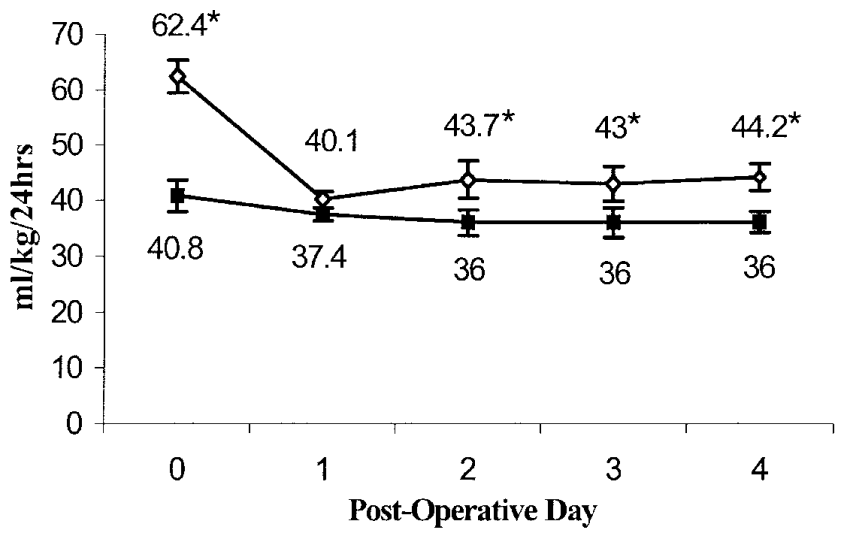

Figure 1. Perioperative urinary output. Open diamonds, The continuous infusion group; filled squares, the bolus dose group. Asterisk indicates $P<.05$.

remaining 75 consecutive patients (Table 1) were randomly divided into two groups according to the diuretic protocol, a group treated with furosemide continuous intravenous therapy $(\mathrm{n}=36)$ and a group treated with bolus furosemide intravenous therapy $(n=39)$.

\section{Diuresis Management}

The target daily urinary output during the first 4 postoperative days was between 25 and $50 \mathrm{~mL} / \mathrm{kg}$, with an emphasis on maintaining negative fluid balance. The first-line diuretic used was furosemide given intravenously for the first 48 to 72 hours as continuous infusion in the continuous infusion group $(2-15 \mathrm{mg} / \mathrm{h})$ and as bolus therapy (50-100 mg bolus) in the bolus dose group. In both groups if the targeted urinary output could not be achieved with furosemide, dopamine at a dopaminergic dose $\left(1-2 \mu \mathrm{g} \cdot \mathrm{kg}^{-1}\right.$ - $\mathrm{min}^{-1}$ ) was added. Spironolactone at $75 \mathrm{mg} / \mathrm{d}$ was orally administered immediately after extubation and maintained for 6 postoperative weeks in both patient groups.

\section{Inotropic Support}

Dobutamine $\left(2-5 \mu \mathrm{g} \cdot \mathrm{kg}^{-1} \cdot \mathrm{min}^{-1}\right)$ was started for all patients during weaning from cardiopulmonary bypass and continued until the second postoperative day. ${ }^{13}$ There was no significant difference between the groups with respect to the total dose of dobutamine used.

\section{Extubation, Oxygen Supplementation, and Reintubation Criteria}

Patients were kept lightly sedated with an intravenous propofol infusion until they fulfilled criteria for extubation. ${ }^{14}$ Supplemental oxygen was given to patients by either nasal cannula or face mask. Patients were supported with oxygen when oxygen saturation could not be maintained above $92 \%$ on room air. Reintubation was instituted when acceptable oxygen saturation could not be maintained despite the use of $100 \%$ oxygen with a nonrebreathing mask or when the respiratory rate to tidal volume (liters) was 90 with subjective shortness of breath, $\mathrm{PaCO}_{2}$ retention $(>50 \mathrm{~mm} \mathrm{Hg})$, and a $\mathrm{pH}$ of 7.3 or lower. ${ }^{15}$

\section{Parameters Checked}

This study was done to assess our new protocol-guided diuresis after the maze procedure. We collected hemodynamic data from all patients' pulmonary artery catheter monitoring during the first 48 hours afer the operation. Urinary outputs, total fluid balances, and the times required to return to preoperative weight were calculated for all patients. Pulmonary complications related to fluid retention were documented and listed in all cases.

\section{Statistical Analysis}

We constructed multivariate models with ordinary least squares linear regression. Intergroup differences were compared using analysis of variance. A 2-tailed Spearman $\rho$ coefficient was calculated to assess the correlation between the dose of furosemide and urinary output. All statistical analyses were performed with the Statistical Package for the Social Sciences version 8.0 (SPSS Inc, Chicago, Ill).

\section{Results}

Patient demographic characteristics were evenly matched between groups with respect to age, sex distribution, baseline creatinine level, cardiopulmonary bypass and crossclamp times, and postoperative cardiac index. A slightly higher percentage of patients in the continuous infusion group underwent a minimally invasive maze procedure; otherwise, both groups underwent similar surgical procedures (Table 1).

\section{Fluid Balance and Urinary Output}

The main concern after the maze procedure is fluid retention. The consequences of fluid overload are mainly pulmonary complications. The vast majority of the patients $(95 \%)$ in the continuous infusion group had negative fluid balance on postoperative days $0,2,3$, and 4 , whereas only $52 \%$ of patients in the bolus dose group did so on postoperative days $0,1,2$, and 3 . There was no difference between the groups with respect to fluid balance on postoperative day 1 . Only on postoperative day 4 did more than $90 \%$ of patients in the bolus dose group have negative fluid balance.

This study correlates with previous studies that have shown the advantage of continuous intravenous furosemide therapy over bolus intravenous furosemide therapy with respect to urinary output. Urinary output was higher in the continuous infusion group patients on every postoperative day and reached statistical significance on postoperative days 0, 2, 3 and 4 (Figure 1). The total dose of furosemide, however, was significantly lower in the continuous infusion group, despite their higher urinary output $(2.8 \pm 2.2 \mathrm{mg} / \mathrm{kg}$ for the continuous infusion group vs $4.7 \pm 2.19 \mathrm{mg} / \mathrm{kg}$ for the bolus dose group, $P<.027)$. Mean daily urinary output was highly influenced by the presence of a continuous furosemide infusion $(P<.001)$. A dose-dependent correlation was observed between the continuous furosemide infusion dose and urinary output (Spearman $\rho=0.43, P<.001$ ).

Multivariate analysis showed that urinary output was positively correlated with performance of a minimally inva- 
sive procedure $(P=.01)$, female sex $(P=.03)$, the presence of a continuous furosemide drip $(P<.0001)$, and the total dose of furosemide $(P=.03)$. Our model, after adjustment for sex and minimally invasive procedures, demonstrated that continuous intravenous furosemide therapy was associated with a significant higher urinary output. Urinary output in this series did not correlate with the complexity of the procedure performed, cardiopulmonary bypass time, crossclamp time, age, postoperative intensive care unit stay, intubation time, and the use of diuretics other than furosemide and spironolactone.

\section{Pulmonary Complications}

Observed pulmonary complications can be divided into two categories, those primarily related to oxygenation or ventilation abnormalities secondary to pulmonary congestion or edema and those related to hypoxemia secondary to pleural effusion. Patients in both categories were treated with additional diuretics. Table 2 charts the results for each group according to the category of pulmonary complications. Small pleural effusions were those with mild blunting of the costophrenic angle without compromised systemic oxygenation, medium were those with moderate costophrenic angle blunting extending anteriorly and some degree of impairment of oxygenation, and large pleural effusions were substantial effusions obliterating the costophrenic angle with a significant impairment of oxygenation. Relative to the continuous infusion group the bolus dose group had a statistically significant higher number of patients with pleural effusions in each subcategory. Greater numbers of the bolus dose group patients required supplemental oxygen therapy, and all reintubations occurred in the bolus dose group. None of the reintubations were for unstable hemodynamic parameters or premature extubation. One patient in the bolus dose group had both pulmonary edema and a large pleural effusion and was treated with diuretics, tube thoracostomy, and reintubation.

\section{Return to Baseline Weight}

Return to preoperative weight occurred more rapidly in the continuous infusion group ( $4 \pm 1.6$ days) than in the bolus dose group $(9.3 \pm 3.5$ days, $P<.036)$. Multivariate analysis showed that the presence of a continuous furosemide drip was correlated with a decreased interval to return to preoperative weight $(P<.001)$. Similarly, an increased dose of furosemide administered was correlated with the patient's weight $(P=.004)$.

\section{Mechanical Ventilation}

The time to initial extubation was greater in the bolus dose group $(13.3 \pm 15.8$ hours $)$ than in the continuous infusion group (6.2 \pm 5.6 hours, $P=.013)$. Multivariate analysis demonstrated that time to extubation was correlated with performance of a minimally invasive procedure $(P=.031)$.
This correlation is important, because the maze procedure is currently done with a minimally invasive approach in more than $70 \%$ of our cases.

\section{Discussion}

Cardiopulmonary bypass for cardiac surgery results in significant fluid accumulation. Longer bypass times lead to greater fluid retention. ${ }^{16-20}$ The maze procedure is a relatively long procedure, especially when combined with valve or coronary artery bypass graft operations. Patients undergoing the maze procedure are thus predisposed toward greater fluid accumulation by of the duration of the operation itself. Additionally, our experience and that of other groups show enhanced fluid retention in patients after the maze procedure relative to other types of cardiac operations. ${ }^{2,3}$ One of the suggested mechanisms is the abrupt decreased postprocedure ANP levels along with cardioversion to sinus rhythm. ${ }^{4,5}$ Recently we showed that both arginine vasopressin and aldosterone circulating levels are high after the maze procedure. Because of these higher levels, patients have higher tendency to retain fluids. Fluid retention and related complications were documented in as many as $30 \%$ of our patients after the maze procedure..$^{2,3}$

Several studies have demonstrated the effectiveness of postoperative furosemide infusion in mitigating fluid retention. ${ }^{6-12}$ Continuous infusions result in a more consistent urinary output, lower urinary loss of electrolytes, and fewer alterations in fluid balance, as reflected by altered cardiac index and filling pressures. ${ }^{7,8,12}$

This study was designed to explore the benefits of protocol-guided diuretic management for patients undergoing the maze procedure and to determine whether continuous and intermittent dosing of furosemide would lead to clinically relevant differences in outcomes. Our results demonstrated several beneficial effects of continuous furosemide infusion relative to intermittent dosing. Total urinary output increased in the continuous infusion group despite a reduction by half of the total dose of furosemide. Continuous infusion did not adversely affect hemodynamics even as it sustained diuresis, and urinary output was easily titrated by changing the infusion rate. Most important, the postoperative course was improved, as reflected in more rapid return to baseline weight, maintenance of negative fluid balance in the immediate postoperative period, and fewer pulmonary complications, especially the absence of reintubation (Table 2). Attainment of negative fluid balance, with its resultant decrease in the time to return to baseline weight, was related to continuous furosemide therapy and to the diminished use of colloid necessary to maintain intravascular volume with bolus furosemide therapy. Negative fluid balance is critical to decreasing pulmonary complications.

We did not find a positive effect of continuous therapy on urinary output or negative fluid balance on postoperative 
day 1 (Figure 1). Interestingly, most of the pulmonary congestion and edema occurred on postoperative day 2 . We surmise that this is because $95 \%$ of patients in the continuous infusion group, versus only $52 \%$ in the bolus dose group, had a negative fluid balance on postoperative day 1 . Earlier negative fluid balance could have protected patients in the bolus dose group from pulmonary complications despite the lack of negative fluid balance that day. The effectiveness of continuous furosemide therapy may be due to the attenuation of the impact of low levels of ANP along with higher levels of aldosterone and arginine vasopressin in patients after the maze procedure.

Minimizing morbidity related to fluid retention did not lead to a decrease in overall hospital stay. It is possible that a return to stable sinus rhythm has a much greater influence on hospital stay than does achievement of negative fluid balance.

Our protocol for all patients after the maze procedure was derived in part from this study. Patients receive continuous infusions of both furosemide $(2-15 \mathrm{mg} / \mathrm{h})$ and dobutamine $\left(2-5 \mu \mathrm{g} \cdot \mathrm{kg}^{-1} \cdot \mathrm{min}^{-1}\right)$ for the first 48 postoperative hours. At the time of extubation, and for 6 weeks afterward, spironolactone (75 $\mathrm{mg}$ orally daily) is added to the diuretic therapy. Spironolactone, initially added because it is a diuretic with a different mechanism of action than furosemide, may also improve overall cardiac performance. ${ }^{21}$ This combined therapeutic regimen has led to significant improvements in the postoperative course of patients after the maze procedure, with or without other cardiac surgical procedures.

In summary, we have demonstrated that continuous furosemide therapy after the maze procedure is a more effective method of achieving higher constant urinary output than intermittent bolus therapy. Continuous furosemide infusion results in significant reductions in fluid accumulation and pulmonary complications.

\section{References}

1. Cox JL. The surgical treatment of atrial fibrillation. IV. Surgical technique. J Thorac Cardiovasc Surg. 1991;101:584-92.

2. Cox JL, Boineau JP, Schuessler RB, Kater KM, Lappas DG. Five-year experience with the maze procedure for atrial fibrillation. Ann Thorac Surg. 1993;56:814-24.

3. McCarthy PM, Cosgrove DM 3rd, Castle LW, White RD, Klein AL. Combined treatment of mitral regurgitation and atrial fibrillation with valvuloplasty and the maze procedure. Am J Cardiol. 1993;71:483-6.

4. Kim $\mathrm{KB}$, Lee $\mathrm{CH}$, Kim $\mathrm{CH}$, Cha YJ. Effect of the Cox maze procedure on the secretion of atrial natriuretic peptide. J Thorac Cardiovasc Surg. 1998;115:139-47.

5. Fujiwara H, Ishikura F, Nagata S, Beppu S, Miyatake K. Plasma atrial natriuretic peptide response to direct current cardioversion of atrial fibrillation in patients with mitral stenosis. $\mathrm{J} \mathrm{Am} \mathrm{Coll} \mathrm{Cardiol.}$ 1993;22:575-80

6. Mookherje S, Anderson G Jr, Smulyan H, Vardan S. Atrial natriuretic peptide response to cardioversion of atrial flutter and fibrillation and the role of associated heart failure. Am J Cardiol. 1991;67:377-80.

7. Coepland JG, Campbell DW, Plachetka JR, Salomon NW, Larson DF. Diuresis with continuous infusion of furosemide after cardiac surgery. Am J Surg. 1983;146:796-9.

8. Magovern JA, Magovern GJ. Diuresis in hemodynamically compromised patients: continuous furosemide infusion. Ann Thorac Surg. 1990; 50:482-4.

9. Martin SJ, Danzinger LH. Continuous infusion of loop diuretics in critically ill: a review of the literature. Crit Care Med. 1994;22:1323-9.

10. Aaser E, Gullestad L, Tollofsrud S, Lundberg J, Hall C, Djoseland O, et al. Effect of bolus injection versus continuous infusion of furosemide on diuresis and neurohormonal activation in patients with severe congestive heart failure. Scand J Clin Lab Invest. 1997;57:361-7.

11. Schuller D, Lynch PJ, Fine D. Protocol-guided diuretic management: comparison of furosemide by continuous infusion and intermitent bolus. Crit Care Med. 1997;25:1969-75.

12. Yelton SL, Gaylor MA, Murray KM. The role of continuous infusion loop diuretics. Ann Pharmacother. 1995;29:1010-4.

13. Akosah KO, Delinger B, Mohanty PK. Safety and hemodynamic responses to beta-adrenergic stimulation by dobutamine in heart transplant patients. Chest. 1999;116:1587-92.

14. Hall JB, Wood LD. Liberation of the patient from mechanical ventilation. JAMA. 1987;257:1621-8.

15. Kirklin JW, Barratt-Boyes BG. Cardiac surgery. New York: Churchill Livingstone; 1993. p. 241.

16. Hakim M, Wheeldon D, Bethune DW, Milstein BB, English TA, Wallwork J. Haemodialysis and haemofiltration on cardiopulmonary bypass. Thorax. 1985;40:101-6.

17. Magilligan DJ. Indications for ultrafiltration in the cardiac surgical patient. J Thorac Cardiovasc Surg. 1985;89:183-9.

18. Elliott MJ. Ultrafiltration and modified ultrafiltration in pediatric open heart operations. Ann Thorac Surg. 1993;56:1518-22.

19. Ungerleider RM. Effects of cardiopulmonary bypass and use of modified ultrafiltration. Ann Thorac Surg. 1998;65(6 Suppl):S35-8.

20. Gonzalez J, Morrissey T, Byrne T, Rizzo R, Wilmore D. Bioelectric impedance detects fluid retention in patients undergoing. J Thorac Cardiovasc Surg. 1995;110:111-8.

21. Pitt B, Zannad F, Remme WJ, Cody R, Castaigne A, Perez A, Palensky J, Witts J. The effect of spironolactone on morbidity and mortality in patients with severe heart failure: Randomized Aldactone Evaluation Study Investigators. N Engl J Med. 1999;341:709-17. 